\title{
Downlink Beamforming Under Individual SINR and Per Antenna Power Constraints
}

\author{
Yakup K. Yazarel and Defne Aktas \\ Dept. of Electrical and Electronics Engineering, Bilkent University, Bilkent, Ankara 06800, Turkey \\ Email: yazarel, daktas @ee.bilkent.edu.tr
}

\begin{abstract}
In this paper we consider the problem of finding the optimum beamforming vectors for the downlink of a multiuser system, where there are individual signal to interference plus noise ratio (SINR) targets for each user. Majority of the previous work on this problem assumed a total power constraint on the base stations. However, since each transmit antenna is limited by the amount of power it can transmit due to the limited linear region of the power amplifiers, a more realistic constraint is to place a limit on the per antenna power. In a recent work, Yu and Lan proposed an iterative algorithm for computing the optimum beamforming vectors minimizing the power margin over al antennas under individual SINR and per antenna power constraints. However, from a system designer point of view, it may be more desirable to minimize the total transmit power rather than minimizing the power margin, especially when the system is not symmetric. Reformulating the transmitter optimization problem to minimize the total transmit power subject to individual SINR constraints on the users and per antenna power constraints on the base stations, the algorithm proposed by $\mathrm{Yu}$ and Lan is modified. Performance of the modified algorithm is compared with existing methods for various cellular array scenarios.
\end{abstract}

\section{INTRODUCTION}

Multiple input multiple output (MIMO) techniques are becoming an essential component of wireless broadband access systems as they can provide high gains in spectral efficiency over single antenna systems by exploiting spatial diversity and multiplexing gains. In order to achieve high spectral efficiencies in the downlink of a multiuser system, the base stations transmit independent data to several mobile users simultaneously by properly adjusting the transmitted signal such that the interference observed at the mobile users is minimized. In fact under a total power constraint, the sum capacity achieving strategy for the MIMO downlink is shown to be a combination of a nonlinear precoding scheme for interference precancellation, known as dirty paper coding (DPC), and transmit beamforming [1]-[3].

We consider the problem of selecting beamforming (BF) vectors given perfect channel state information at the base stations such that individual signal to interference plus noise ratio (SINR) targets are achieved at the mobile users. Significant progress has recently been made on this problem. In [4], an iterative algorithm to compute the optimal power allocation and BF vectors maximizing the minimum SINR margin subject to a total transmit power constraint is proposed. Furthermore, it is shown that this algorithm can be modified to determine the optimal transmission strategy minimizing the total transmit power subject to individual SINR constraints. The algorithm in [4] is later extended to the case where base stations employ DPC together with BF to further reduce the interference at the users [5]. A fast iterative algorithm extending the results in [4] to the case where beamforming vectors are under a quadratic constraint is provided in [6]. Wiesel et al. provided an alternative simple and iterative algorithm based on reformulating the original nonconvex optimization problem as a second order cone programming problem in [7].

This work was supported by the European Commission Sixth Framework Programme Marie Curie International Reintegration Grant MIRG-CT-2005029179 .
Majority of the previous work on downlink BF with individual SINR constraints assume a total transmit power constraint. However, in practice, each transmit antenna is limited in the amount of power that it can transmit due to the limited linear range of its power amplifier. Therefore, it is more realistic to consider the transmitter optimization problem under a per antenna power constraint. Per antenna power constraint is also required for cooperative downlink systems, where the base stations cooperate in transmitting information to users in the system, as each cooperating base station will have its own power limitation on its antennas [8]. Optimum power allocation using zero-forcing (ZF) beamformers under per antenna power constraints has been investigated in [8], [9]. However, ZF beamformers are known to be suboptimal especially when the signal to noise ratio (SNR) and the number of users are not sufficiently large due excessive use of power to null out the interference.

In a recent work, Yu and Lan proposed an efficient iterative method for computing the BF vectors minimizing the power margin over all antennas under individual SINR constraints [10]. In the same work, an interior point method for characterizing the achievable rate region under per antenna power constraints is provided. However, the algorithm for computing the $\mathrm{BF}$ vectors minimizing the power margin over all antennas assumes that the given set of SINR constraints are feasible. Therefore, the algorithm may give unpredictable results when the SINR constraints are not feasible. Furthermore, minimizing the total power subject to individual SINR and per antenna power constraints may be more desirable than minimizing the power margin over all antennas from the system designer point of view, especially when the system is not symmetric.

In this work, we reformulate the transmitter optimization problem in [10] as finding the BF vectors minimizing the total transmit power under individual SINR constraints at the users and per antenna power constraints at the base stations. Following the Lagrangian duality formulation, we modified the iterative algorithm in [10] to compute $\mathrm{BF}$ vectors minimizing the total transmit power. We compared the per antenna power consumption of the modified algorithm to existing methods for different cellular array configurations.

\section{SySTEM MODEL}

The notation used in the paper is as follows. Boldface letters is used to denote vectors and matrices. For a given matrix $\mathbf{A}, \mathbf{A}^{T}, \mathbf{A}^{H}, \mathbf{A}^{-1}$, $\operatorname{tr}(\mathbf{A})$ and $\mathbf{A}_{i j}$ denote the transpose, the conjugate transpose, the inverse, the trace and the $(i, j)$ th element of $\mathbf{A}$, respectively. I denotes the identity matrix and $\operatorname{diag}(\mathbf{b})$ denotes a diagonal matrix whose $i$ th diagonal element is $\mathbf{b}_{i}$. The expectation operation is denoted as $E[\cdot]$.

We consider a multiuser system where a base station with $N$ antennas is transmitting independent information to $K$ users, each equipped with a single antenna. We assume that the channel is a frequency flat fading channel and is known at both the base station and the users. Let $\mathbf{x}=\left[x_{1}, \ldots, x_{N}\right]^{T}$ denote the transmit signal vector and $\mathbf{h}_{i}^{H}$ denote the $1 \times N$ channel vector consisting of channel 
gain between $i$ th user and the base station antennas. The noise at $i$ th user, $n_{i}$, is modeled as circularly symmetric complex Gaussian random variable with zero mean and variance $\sigma^{2}$. The received signal at the user $i$ is then given by

$$
y_{i}=\mathbf{h}_{i}^{H} \mathbf{x}+n_{i} .
$$

Since the base station is using beamforming, the information for the users is mapped to the transmitted signal by

$$
\mathbf{x}=\sum_{k=1}^{K} s_{k} \mathbf{w}_{k}
$$

where $s_{k}$ is the normalized information symbol for user $k$ with $E\left[\left|s_{k}\right|^{2}\right]=1$ and $\mathbf{w}_{k}$ is the beamforming vector for user $k$. Note that with this formulation, the power allocation for the $k$ th user is given as $\tilde{P}_{k}=\mathbf{w}_{k}^{H} \mathbf{w}_{k}$. The power consumption for the $n$th base station antenna is given by $\hat{P}_{n}=E\left[\left|x_{n}\right|^{2}\right]=\left(\sum_{j=1}^{K} \mathbf{w}_{j} \mathbf{w}_{j}^{H}\right)_{n n}$.

Assuming that the users do not cooperate and do not employ sophisticated interference cancellation or suppression techniques, the interference from other users will be treated as additional noise and the signal to interference plus noise ratio (SINR) for user $i$ given by

$$
\operatorname{SINR}_{i}=\frac{\left|\mathbf{h}_{i}^{H} \mathbf{w}_{i}\right|^{2}}{\sum_{\substack{k=1 \\ k \neq i}}^{K}\left|\mathbf{h}_{i}^{H} \mathbf{w}_{k}\right|^{2}+\sigma^{2}}
$$

can be used as a performance metric.

\section{Optimal BEAMForming WITH SINR AND PER ANTENNA POWER CONSTRAINTS}

The problem of interest is to determine the optimal BF vectors minimizing the total transmit power subject to individual SINR requirements on the users corresponding to possibly different QoS requirements and per antenna power constraints. The optimization problem can be stated as

$$
\begin{aligned}
\underset{\mathbf{w}_{1}, \ldots, \mathbf{w}_{K}}{\operatorname{minimize}} & \sum_{k=1}^{K} \mathbf{w}_{k}^{H} \mathbf{w}_{k} \\
\text { subject to } & \left(\sum_{j=1}^{K} \mathbf{w}_{j} \mathbf{w}_{j}^{H}\right)_{n n} \leq P_{n} \quad n=1, \ldots, N, \\
& \frac{\left|\mathbf{h}_{i}^{H} \mathbf{w}_{i}\right|^{2}}{\sum_{k \neq i}\left|\mathbf{h}_{i}^{H} \mathbf{w}_{k}\right|^{2}+\sigma^{2}} \geq \gamma_{i} \quad i=1, \ldots, K,
\end{aligned}
$$

where $\gamma_{1}, \ldots, \gamma_{K}$ are the target SINRs and $P_{1}, \ldots, P_{N}$ are the per antenna power requirements.

$\mathrm{Yu}$ and Lan considered the following related problem and proposed an elegant iterative algorithm based on Lagrangian dual problem:

$$
\begin{aligned}
\underset{\text { w }_{1}, \ldots, \mathrm{w}_{K}, \alpha}{\operatorname{minimize}} \alpha & \\
\text { subject to } & \left(\sum_{j=1}^{K} \mathbf{w}_{j} \mathbf{w}_{j}^{H}\right)_{n n} \leq \alpha P_{n} \quad \forall n, \\
& \frac{\left|\mathbf{h}_{i}^{H} \mathbf{w}_{i}\right|^{2}}{\sum_{k \neq i}\left|\mathbf{h}_{i}^{H} \mathbf{w}_{k}\right|^{2}+\sigma^{2}} \geq \gamma_{i} \quad \forall i,
\end{aligned}
$$

which is aiming to minimize the maximum power margin $\hat{P}_{i} / P_{i}$ over all antennas [10]. From a system designer point of view, minimizing the total power is more critical than minimizing the power margin, especially when the system is not symmetric. Note that since there is no constraint on $\alpha$ to satisfy $\alpha \leq 1$, if the problem is infeasible, the algorithm will result in $\alpha>1$ violating the power constraints.

In this section, using the Lagrangian duality framework, we will modify the iterative algorithm in [10] to find the optimum BF vectors for the original problem stated in (4)-(6). Note that the original problem is not convex, however as noted in [10], it can be stated as a second-order cone program (SOCP) by restricting $\mathbf{h}_{k}^{H} \mathbf{w}_{k}$ to be real valued. As a result, strong duality holds and we can solve the dual problem.

The Lagrangian function of the SOCP has the same form as the Lagrangian function of the original problem given by

$$
\begin{aligned}
L(\mathbf{W}, \mathbf{Q}, \lambda) & =\sum_{i=1}^{K} \lambda_{i} \sigma^{2}-\operatorname{tr}(\mathbf{Q} \Phi) \\
& +\sum_{k=1}^{K} \mathbf{w}_{k}^{H}\left(\mathbf{Q}+\mathbf{I}+\sum_{j \neq k} \lambda_{j} \mathbf{h}_{j} \mathbf{h}_{j}^{H}-\frac{\lambda_{k}}{\gamma_{k}} \mathbf{h}_{k} \mathbf{h}_{k}^{H}\right) \mathbf{w}_{k},
\end{aligned}
$$

where $\mathbf{Q}=\operatorname{diag}\left(\left[q_{1}, \ldots, q_{N}\right]\right)$ and $\lambda=\left[\lambda_{1}, \ldots, \lambda_{K}\right]$ are the matrices of dual variables for the antenna power and the SINR constraints, respectively and $\boldsymbol{\Phi}=\operatorname{diag}\left(\left[P_{1}, \ldots, P_{N}\right]\right)$ and $\mathbf{W}=$ $\left[\mathbf{w}_{1}, \ldots, \mathbf{w}_{K}\right]$. The dual function is therefore given by

$$
g(\mathbf{Q}, \lambda)=\min _{\mathrm{w}_{1}, \ldots, \mathrm{w}} L(\mathbf{W}, \mathbf{Q}, \lambda)
$$

which is unbounded unless $\mathbf{Q}+\mathbf{I}+\sum_{j \neq k} \lambda_{j} \mathbf{h}_{j} \mathbf{h}_{j}^{H}-\frac{\lambda_{k}}{\gamma_{k}} \mathbf{h}_{k} \mathbf{h}_{k}^{H}$ is positive semidefinite [10].

Defining

$$
\hat{\mathbf{w}}_{k}=\left(\mathbf{I}+\mathbf{Q}+\sum_{j=1}^{K} \lambda_{j} \mathbf{h}_{j} \mathbf{h}_{j}^{H}\right)^{\dagger} \mathbf{h}_{k},
$$

where $(.)^{\dagger}$ denotes the matrix pseudoinverse, for given $\mathbf{Q}$ and $\lambda$, the optimum $\mathbf{w}_{k}$ minimizing (11) satisfies

$$
\mathbf{w}_{k}=\hat{\mathbf{w}}_{k}\left(1+\frac{1}{\gamma_{k}}\right) \lambda_{k} \mathbf{h}_{k}^{H} \mathbf{w}_{k}
$$

Since the optimum $\mathbf{w}_{k}$ makes the term with BF vectors in (10) zero, the dual variables corresponding to SINR constraints satisfy the following set of fixed point equations

$$
\lambda_{k}=\frac{1}{\left(1+\frac{1}{\gamma_{k}}\right) \mathbf{h}_{k}^{H}\left(\mathbf{I}+\mathbf{Q}+\sum_{j=1}^{K} \lambda_{j} \mathbf{h}_{j} \mathbf{h}_{j}^{H}\right)^{\dagger} \mathbf{h}_{k}},
$$

for all $k$. If the original problem is feasible, then the SINR constraints should be met with equality. Therefore, as in [10], defining $\mathbf{w}_{k}=$ $\sqrt{\delta_{k}} \hat{\mathbf{w}}_{k}$, the scaling factors satisfy $\left[\delta_{1}, \ldots, \delta_{K}\right]^{T}=\mathbf{G}^{-1} \mathbf{1} \sigma^{2}$, where $\mathbf{1}$ denotes an all ones vector, $\mathbf{G}_{i i}=\frac{1}{\gamma_{i}}\left|\hat{\mathbf{w}}_{i}^{H} \mathbf{h}_{i}\right|^{2}$ and $\mathbf{G}_{i j}=$ $-\left|\hat{\mathbf{w}}_{j}^{H} \mathbf{h}_{i}\right|^{2}$.

The Lagrange dual problem is then stated as

$$
\max _{Q} \sum_{k=1}^{K} \lambda_{k} \sigma^{2}-\operatorname{tr}(\mathbf{Q \Phi})
$$

subject to

$$
\begin{gathered}
\lambda_{k}=\frac{1}{\left(1+\frac{1}{\gamma_{k}}\right) \mathbf{h}_{k}^{H}\left(\mathbf{I}+\mathbf{Q}+\sum_{j=1}^{K} \lambda_{j} \mathbf{h}_{j} \mathbf{h}_{j}^{H}\right)^{\dagger} \mathbf{h}_{k}}, \quad \forall k \\
\mathbf{Q}: \text { diagonal, } \quad \mathbf{Q} \succeq 0 .
\end{gathered}
$$


The argument of the maximization problem in (15) can be shown to be concave in $\mathbf{Q}$ with a subgradient $\operatorname{diag}(\mathbf{b})-\boldsymbol{\Phi}$, where $\mathbf{b}_{n}=$ $\left(\sum_{k=1}^{K} \mathbf{w}_{k} \mathbf{w}_{k}^{H}\right)_{n n}$, following a similar approach as in [10].

Hence the iterative algorithm in [10] is modified as follows to solve (4)-(6).

1) Initialize: $t \leftarrow 0, \mathbf{Q}^{(0)}:$ diagonal.

2) $t \leftarrow t+1$.

3) Solve (14) for $\lambda^{(t)}$ with $\mathbf{Q} \leftarrow \mathbf{Q}^{(t-1)}$ using fixed point iteration method.

4) Compute $\hat{\mathbf{w}}_{k}^{(t)}$ using (12) with $\mathbf{Q} \leftarrow \mathbf{Q}^{(t-1)}$ and $\lambda_{k} \leftarrow \lambda_{k}^{(t)}$.

5) Compute the BF vectors as $\mathbf{w}_{k}^{(t)}=\sqrt{\delta_{k}} \hat{\mathbf{w}}_{k}^{(t)}$ where $\delta_{k}=$ $\sigma^{2} \sum_{j=1}^{K}\left(\mathbf{G}^{-1}\right)_{k j}$ with $\mathbf{G}_{i i}=\frac{1}{\gamma_{i}}\left|\mathbf{h}_{i}^{H} \hat{\mathbf{w}}_{i}^{(t)}\right|^{2}$ and $\mathbf{G}_{i j}=$ $-\left|\mathbf{h}_{i}^{H} \hat{\mathbf{w}}_{j}^{(t)}\right|^{2}$.

6) Update $\mathbf{Q}^{(t)}$ as

$$
q_{n}^{(t)}=\max \left(0, q_{n}^{(t-1)}+\mu_{t}\left(\operatorname{diag}\left(\mathbf{b}^{(t)}\right)-\mathbf{\Phi}\right)\right)
$$

where $\mathbf{b}_{n}^{(t)}=\left(\sum_{k=1}^{K} \mathbf{w}_{k}^{(t)}\left(\mathbf{w}_{k}^{(t)}\right)^{H}\right)$ and $\mu_{t}$ is the step size.

7) Go to Step 2 if the algorithm did not converge.

Since strong duality holds, the nonconvex optimization problem in (4) can be solved globally by the convex dual problem by the algorithm given above. One can easily show that the fixed point iteration converges, as the derivative of the iteration function has norm less than 1. Similarly, one can show that the subgradient projection algorithm for finding the optimum $\mathbf{Q}$ converges [10].

Note that as stated in [10], one can also incorporate the case where the base station utilizes DPC with encoding order $\pi(1), \ldots, \pi(K)$. In this case, the SINR for user $\pi(i)$ is given as

$$
\operatorname{SINR}_{\pi(i)}=\frac{\left|\mathbf{h}_{\pi(i)}^{H} \mathbf{w}_{\pi(i)}\right|^{2}}{\sum_{k=i+1}^{K}\left|\mathbf{h}_{\pi(i)}^{H} \mathbf{w}_{\pi(k)}\right|^{2}+\sigma^{2}}
$$

With DPC, the algorithm is modified as follows. The vector in (12) is now computed as

$$
\hat{\mathbf{w}}_{\pi(k)}=\left(\mathbf{I}+\mathbf{Q}+\sum_{j=1}^{k} \lambda_{\pi(j)} \mathbf{h}_{\pi(j)} \mathbf{h}_{\pi(j)}^{H}\right)^{\dagger} \mathbf{h}_{\pi(k)} .
$$

The optimal BF vectors are given as $\mathbf{w}_{\pi(k)}=\sqrt{\delta_{\pi(k)}} \hat{\mathbf{w}}_{\pi(k)}$, where $\left[\delta_{\pi(1)}, \ldots, \delta_{\pi(K)}\right]^{T}=\mathbf{G}^{-1} \mathbf{1} \sigma^{2}$ with the upper diagonal matrix $\mathbf{G}$ computed as

$$
\mathbf{G}_{i j}= \begin{cases}\frac{1}{\gamma_{\pi(i)}}\left|\hat{\mathbf{w}}_{\pi(i)}^{H} \mathbf{h}_{\pi(i)}\right|^{2}, & i=j \\ -\left|\hat{\mathbf{w}}_{\pi(j)}^{H} \mathbf{h}_{\pi(i)}\right|^{2}, & j>i \\ 0, & \text { otherwise. }\end{cases}
$$

Finally, the fixed point equation in (14) is modified as

$$
\begin{aligned}
\lambda_{\pi(k)} & =\left(\frac{\gamma_{\pi(k)}}{1+\gamma_{\pi(k)}}\right) \\
& {\left[\mathbf{h}_{\pi(k)}^{H}\left(\mathbf{I}+\mathbf{Q}+\sum_{j=1}^{k} \lambda_{\pi(j)} \mathbf{h}_{\pi(j)} \mathbf{h}_{\pi(j)}^{H}\right)^{\dagger} \mathbf{h}_{\pi(k)}\right]^{-1} }
\end{aligned}
$$

\section{NumericAl RESUlts}

Even though the algorithm presented in Section III is general for an arbitrary channel, in order to provide insight we consider a simple cellular network model inspired from [11], where $N$ cells are arranged on a circle. Users in each cell are served by a single

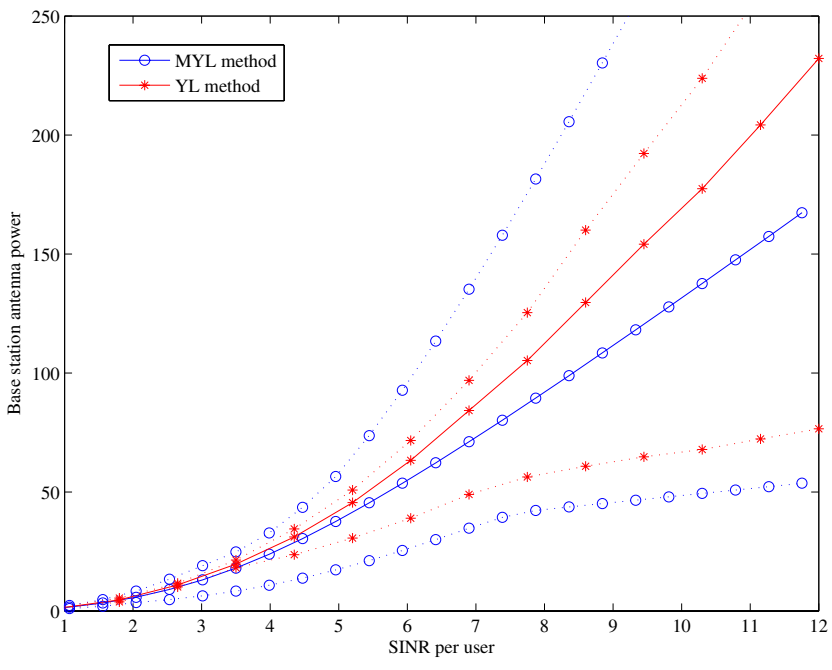

Fig. 1. Comparison of MYL and YL method for an asymmetric circular cellular array. Average BS antenna powers are plotted with solid lines and the minimum (maximum) BS antenna powers are plotted with dotted lines.

antenna base station (BS) located in the middle of the cell and due to an orthogonal multiple access scheme, each BS serves only one user in its cell at a given time/frequency/code. The users are assumed to be equipped with a single antenna and receive signals from three closest base stations only. The entries of the channel matrix $\mathbf{H}$ is modeled as

$$
\mathbf{H}_{i j}= \begin{cases}1, & i=j \\ \alpha_{j}^{f} & i=\bmod (j+1, N) \\ \alpha_{j}^{b} & i=\bmod (j-1, N) \\ 0 & \text { otherwise }\end{cases}
$$

where $\alpha_{j}^{f}, \alpha_{j}^{b} \in[0,1]$ and determine the level of interference from a BS to users in two immediate neighboring cells. We assume that BSs are connected to each other by a high capacity backbone and cooperate in transmission of information to the users.

We first consider an asymmetric circular cellular array scenario with $N=10$ cells, where $\alpha_{j}^{f}$ and $\alpha_{j}^{b}$ are randomly chosen. Figure 1 compares the BS antenna power as a function of a common target SINR for the users computed using the algorithm presented in Section III denoted as MYL and the algorithm in [10] denoted as YL. The solid lines represent the average BS antenna power and the dotted lines represent the minimum (maximum) BS antenna power over the antennas. MYL algorithm computes the BF vectors minimizing the total transmit power subject to SINR and per BS antenna power constraints and YL algorithm minimizes the power margin over all BS antennas under the same constraints. As seen in the figure, MYL algorithm results in a lower total transmit power, especially for high SINR targets, but BS antenna powers have higher variation. On the contrary, YL algorithm results in less variance since the algorithm balances the power margin over the antennas, but the total transmit power is higher.

In [8], an algorithm that computes the optimum user power allocation with zero forcing (ZF) BF vectors maximizing the minimum rate over the users subject to per antenna power constraints is proposed. The performance of the modified YL algorithm is compared with that of the $\mathrm{ZF}$ BF for a symmetric circular cellular array with $\alpha_{j}^{f}=\alpha_{j}^{b}=\alpha, \forall j$ and $N=5$ in Figure 2. The average BS antenna power is plotted as a function of SINR achieved at the users 


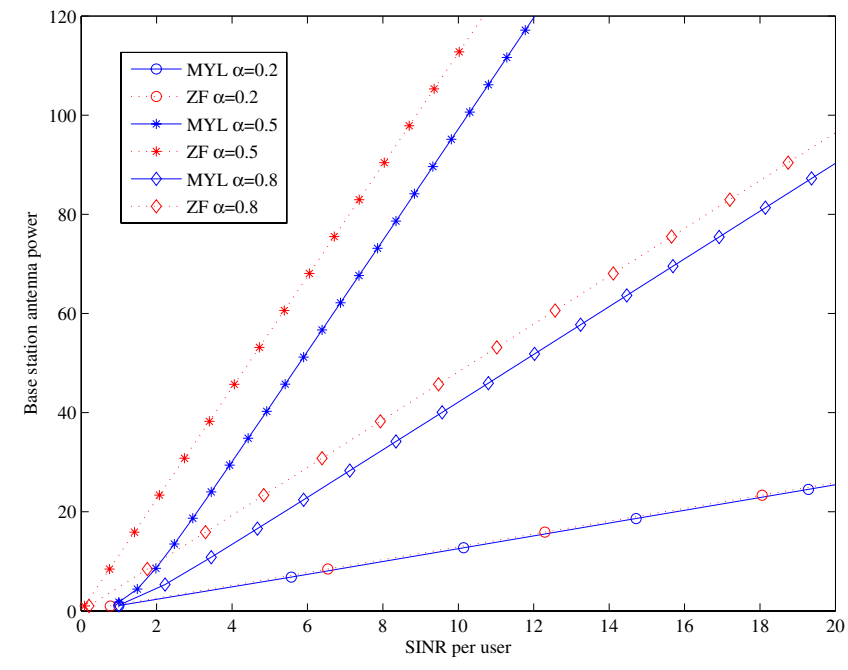

Fig. 2. Comparison of the average BS antenna power using optimum BF vectors (MYL) and zero forcing (ZF) BF vectors for a symmetric circular cellular array with different $\alpha$ and $N=5$.

for various $\alpha$. When the interference is low, i.e. $\alpha$ is small, the total transmit power with both approaches are the same. However, when the interference is high, to achieve the same target SINR at the users $\mathrm{ZF}$ beamforming requires higher total transmit power. This is as expected since nulling the interference completely requires excessive use of the transmit power.

The sum capacity achieving transmission scheme is a combination of beamforming with dirty paper coding (DPC), a successive encoding scheme for precancelling the interference [1]-[3]. To compare the BS antenna power consumption with a downlink transmission scheme employing beamforming only and a scheme employing beamforming with DPC, we considered a symmetric circular array scenario with $N=15$ and $\alpha=0.5$. In Figure 3 the BS antenna power consumption of the two transmission schemes computed using the algorithm presented in Section III are presented. Solid lines denote the average BS antenna power and dotted lines denote the minimum (maximum) BS antenna power. The curve marked with $\circ$ indicates the transmission scheme with BF only and the curves marked with $*$ indicate the results for the scheme using BF with DPC. As expected, to achieve the same target SINR at the users, BF only scheme uses higher BS antenna power than that for BF with DPC using encoding order $\pi=[1, \ldots, N]$. Since the channel is symmetric, the transmit power from all BS antennas are equal for the BF only case. However, since DPC encoding introduces asymmetry to the problem, there is a high variation in the BS antenna power for the BF with DPC scheme, especially for high target SINR values.

\section{Conclusions}

We consider the problem of finding the optimal beamforming vectors for the downlink of a wireless network minimizing the total transmit power subject to individual SINR constraints at the users and per antenna power constraints at the base stations. Per BS antenna power constraints are more realistic as each antenna is limited by the linear range of its power amplifier. Furthermore, per BS antenna power constraints are also required for macrodiversity systems where the base stations cooperate in transmission of the information to the users. In this case the antennas of the super-transmitter are located in separate base stations each with their own power limitations. This problem was investigated in [10], however the optimization criteria was chosen as minimizing the power margin over all antennas. As

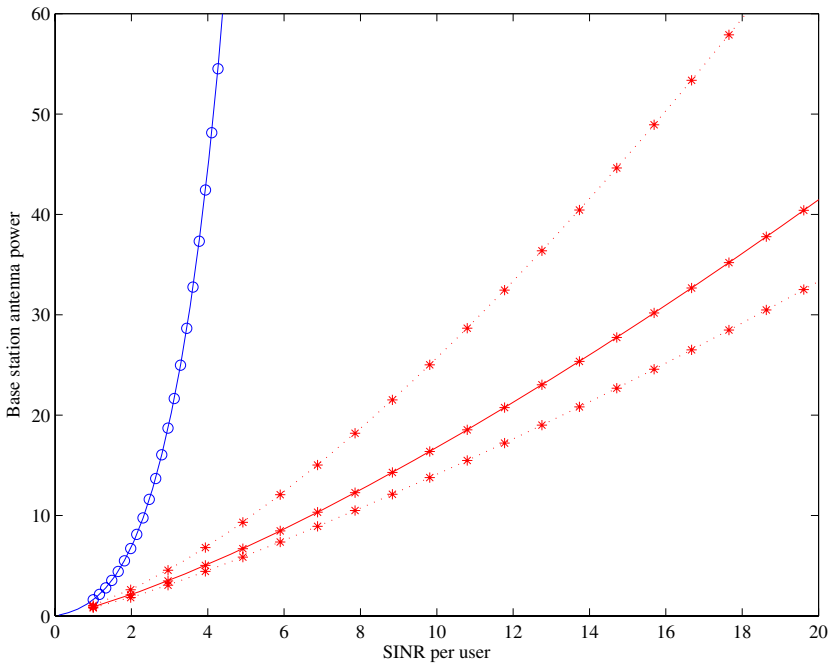

Fig. 3. Comparison of the BS antenna power consumption of the transmission scheme utilizing BF only and the scheme utilizing DPC with BF for a symmetric circular cellular array with $N=15$ and $\alpha=0.5$. Curve marked with $\circ$ indicates BF only and the curves with $*$ indicate BF with DPC schemes. Solid lines denote the average BS antenna power and the dotted lines denote the minimum (maximum) BS antenna power.

illustrated in Section IV, this approach might result in excessive power consumption, especially when the system is asymmetric. Using the Lagrangian duality formulation, we modified the algorithm in [10] to compute the optimum $\mathrm{BF}$ vectors minimizing the total transmit power. Performance of the proposed algorithm is compared with the existing methods. It is observed that the computational complexity of the proposed algorithm is lower than that of [10].

\section{REFERENCES}

[1] P. Viswanath and D. N. C. Tse, "Sum capacity of the vector Gaussian broadcast channel and uplink-downlink duality," IEEE Trans. Inform. Theory, vol. 49, no. 9, pp. 1912-1921, Aug. 2003.

[2] S. Vishwanath, N. Jindal, and A. Goldsmith, "Duality, achievable rates, and sum-rate capacity of Gaussian MIMO broadcast channels," IEEE Trans. Inform. Theory, vol. 49, no. 10, pp. 2658-2668, Oct. 2003.

[3] W. Yu and J. M. Cioffi, "Sum capacity of Gaussian vector broadcast channels," IEEE Trans. Inform. Theory, vol. 50, no. 9, pp. 1875-1892, Sept. 2004.

[4] M. Schubert and H. Boche, "Solution of the multiuser downlink beamforming problem with individual SINR constraints," IEEE Trans. Veh. Technol., vol. 53, no. 1, pp. 18-28, Jan. 2004.

[5] _ , "Iterative multiuser uplink and downlink beamforming under SINR constraints," IEEE Trans. Signal Processing, vol. 53, no. 7, pp. 2324-2334, July 2005.

[6] D. Samuelsson, M. Bengtsson, and B. Ottersten, "An efficient algorithm for solving the downlink beamforming problem with indefinite constraints," in Proc. IEEE Int. Conf. Acoustics, Speech and Signal Processing (ICASSP), vol. 4, Philadelphia, PA, Mar. 2005, pp. 905-908.

[7] A. Wiesel, Y. C. Eldar, and S. Shamai, "Linear precoding via conic optimization for fixed MIMO receivers," IEEE Trans. Signal Processing, vol. 54 , no. 1 , pp. 161-176, Jan. 2006

[8] G. J. Foschini, K. Karakayali, and R. A. Valenzuela, "Coordinating multiple antenna cellular networks to achieve enormous spectral efficiency," IEE Proc. Commun., vol. 153, no. 4, pp. 548-555, Aug. 2006.

[9] F. Boccardi and H. Huang, "Optimum power allocation for the MIMOBC zero-forcing precoder with per-antenna power constraints," in Proc. Annual Conf. Inform. Sciences and Systems (CISS), Princeton, NJ, Mar. 2006, p. 504.

[10] W. Yu and T. Lan, "Transmitter optimization for the multi-antenna downlink with per-antenna power constraints," IEEE Trans. Signal Processing, 2007, to be published in.

[11] A. D. Wyner, "Shannon-theoretic approach to a Gaussian cellular multiple access channel," IEEE Trans. Inform. Theory, vol. 40, no. 6, pp. 1713-1727, Nov. 1994. 